https://doi.org/10.22319/rmcp.v12i1.5273

Artículo

\title{
Evaluación de indicadores productivos en rebaños caprinos vacunados con cepas RB51-SOD, RB51 (Brucella abortus) y Rev-1 (Brucella melitensis)
}

Baldomero Molina-Sánchez ${ }^{\text {a }}$

David Izcoatl Martínez-Herrera ${ }^{a *}$

Violeta Trinidad Pardío-Sedas ${ }^{\text {a }}$

Ricardo Flores-Castro ${ }^{b}$

José A. Villagómez-Cortés ${ }^{\text {a }}$

José F. Morales-Álvarez ${ }^{\mathrm{c}}$

${ }^{\text {a } U n i v e r s i d a d ~ V e r a c r u z a n a . ~ F a c u l t a d ~ d e ~ M e d i c i n a ~ V e t e r i n a r i a ~ y ~ Z o o t e c n i a . ~ A v . ~ M i g u e l ~ A ́ n g e l ~}$ de Quevedo s/n, esq. Yáñez, Col. Unidad Veracruzana, 91710, Veracruz, Veracruz, México.

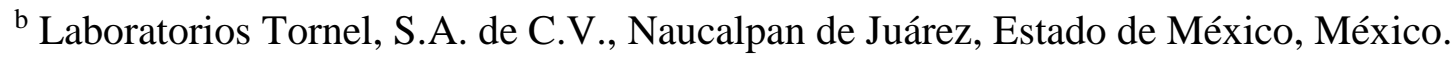

${ }^{\mathrm{c}}$ Instituto Nacional de Investigaciones Forestales, Agrícolas y Pecuarias. Centro Nacional de Investigación Disciplinaria en Salud Animal e Inocuidad (CENID-SAI), Ciudad de México, México.

*Autor de correspondencia: dmartinez@uv.mx

\section{Resumen:}

Se determinaron tasas de pariciones, abortos y nacimiento de crías débiles en rebaños vacunados con la cepa RB51-SOD (B. abortus) para evaluar la mejora productiva y compararla con las vacunas Rev-1 (B. melitensis) y RB51 (B. abortus). Se vacunaron tres

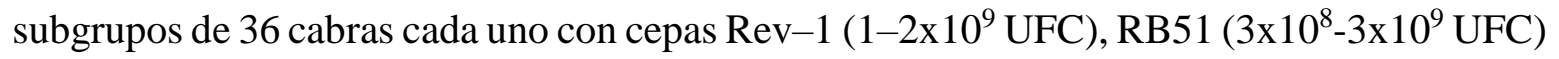
y RB51-SOD ( $3 \times 10^{8}-3 \times 10^{9}$ UFC), cada cepa tuvo un subgrupo control. Se establecieron registros individuales para calcular las tasas posvacunación en dos épocas de pariciones. En 
la primera, la tasa de partos para Rev-1 fue 66.6 \% (IC95\%: 48.9-80.9), RB51 50.0 \% (IC95\% :33.2-66.7) y RB51-SOD $69.4 \%$ (IC95\% :51.7-83.0). Los abortos se presentaron en los tres subgrupos vacunados, Rev-1 y RB51-SOD $5.5 \%$ (IC95\%:0.9-20.0) y RB51 $2.7 \%$ (IC95\%: 0.1-16.2). Los nacimientos de crías débiles se presentaron solo en animales vacunados con Rev-1 $5.5 \%$ (IC95\%: 0.9-20.0). Durante la segunda época, la tasa de partos en hembras vacunadas con Rev-1 fue de $91.6 \%$ (IC95\% :76.4-97.8), RB51 $94.4 \%$ (IC95\% :79.9-99.0) y RB51-SOD 94.4\% (IC95\% :79.9-99.0). Tuvieron abortos animales vacunados con cepas Rev-1 y RB51, $5.5 \%$ (IC95\%: 0.9-20.0) y $2.7 \%$ (IC95\%: 0.1-16.2), respectivamente; en subgrupos vacunados no hubo nacimiento de crías débiles. Los subgrupos controles tuvieron un comportamiento similar a los vacunados. Los animales vacunados con la cepa RB51-SOD no mostraron diferencia significativa con los que recibieron las cepas Rev-1 y RB51, ni con los subgrupos control ( $P>0.01)$; por lo anterior, la vacuna RB51-SOD puede generar protección contra la brucelosis y beneficios en la producción de rebaños caprinos.

Palabras clave: Vacunación, Abortos, Brucelosis, Cabras, RB51- SOD.

Recibido: 21/02/2019

Aceptado: 13/12/2019

\section{Introducción}

La brucelosis es una enfermedad emergente y de distribución mundial que se considera entre las 10 zoonosis olvidadas por las autoridades sanitarias ${ }^{(1,2)}$. Desde el punto de vista económico, reviste importancia por las afectaciones que provoca en las unidades de producción animal, así como por el riesgo para la población humana ${ }^{(3)}$. Los productores de cabras manifiestan que la actividad presenta rezagos tecnológicos y sanitarios, y destacan la persistencia de la brucelosis caprina, misma que merma la productividad, reduce la calidad de la leche y representa un riesgo de infección al hombre ${ }^{(4)}$. La enfermedad es causada por bacterias del género Brucella; las especies más virulentas son Brucella melitensis y Brucella abortus, responsables de la enfermedad en pequeños rumiantes y ganado vacuno, respectivamente $^{(5)}$. La manifestación clínica de la infección en los animales gestantes incluye: aborto, nacimiento de crías que mueren en el periparto y artritis ${ }^{(6,7)}$. En los rebaños caprinos afectados, se aprecia una baja eficiencia productiva por la infertilidad que provoca en animales infectados; los abortos se incrementan hasta un $20 \%$ y disminuye la capacidad productiva en hembras enfermas hasta en $30 \%{ }^{(8,9)}$. 
La baja tasa de parición es el resultado de abortos que ocurren debido a las condiciones sanitarias, entre ellas la prevalencia persistente de la brucelosis y la severa restricción nutricional durante la gestación ${ }^{(10-13)}$ En los rebaños infectados por brucelosis, la vacunación, el diagnóstico y el sacrificio selectivo de animales, son alternativas para el control o erradicación de la enfermedad $^{(2)}$. En la actualidad la cepa Rev-1 de Brucella melitensis, es una cepa viva modificada que se utiliza para controlar la infección en ovinos y caprinos; no obstante, tiene limitantes como la capacidad para inducir aborto en hembras gestantes, excretarse en leche, infectar a los humanos y su posible resistencia a la estreptomicina, un antibiótico que, en combinación con la doxiciclina, constituye el tratamiento más efectivo para la brucelosis en humanos ${ }^{(12,14,15)}$.

La cepa RB51 de Bruella abortus se utiliza para el control de la brucelosis en bovinos y ha sido evaluada en pequeños rumiantes en condiciones controladas con buena protección contra el desafío experimental con B. melitensis $^{(14)}$. Existe información que sostiene que la protección conferida es menor que la obtenida con la cepa Rev-1 y que causa abortos y mortinatos en caprinos ${ }^{(14)}$; sin embargo, tiene la ventaja de no producir interferencia diagnóstica posvacunal frente a la serología convencional ${ }^{(16,17,18)}$.

Las vacunas de plásmidos de ADN tienen potencial para ser el futuro para el control de la brucelosis, se han evaluado cepas de sobreexpresión homóloga para inducir una respuesta inmunitaria. Se ha demostrado que la sobreexpresión de Cu/Zn SOD (superóxido dismutasa), que es una proteína periplásmica que ha desarrollado protección en modelos murinos, frente a la infección experimental con B. abortus cepa virulenta 2308 consigue una protección igual a la inducida por RB51 $(\text { B. abortus })^{(16,19)}$. Oñate at al ${ }^{(19)}$ evaluaron la cepa SOD en bovinos y obtuvieron respuesta de anticuerpos y CMI de tipo Th-1, y protección al desafío de $B$. abortus. Se requieren otros estudios para conocer el papel de diferentes tipos de células T en la protección inducida por vacunación con pcDNA-SOD y sus resultados en sistemas productivos $^{(19-22)}$. La respuesta inmunológica y la eficacia de las vacunas pueden diferir entre los animales de laboratorio y los rumiantes susceptibles ${ }^{(22)}$. Debido a que se carece de información sobre el uso de la cepa RB51 - SOD en animales domésticos, sus efectos y beneficios, así como su seguridad para evitar la inducción de aborto por efecto vacunal y la protección para la mejora productiva en rebaños caprinos, el objetivo de este estudio fue determinar las tasas de pariciones, abortos y nacimiento de crías débiles en rebaños vacunados con la cepa RB51-SOD (Brucella abortus) para evaluar la mejora productiva y compararla con la obtenida al utilizar las vacunas Rev-1 (Brucella melitensis) y RB51 (Brucella abortus). 


\section{Material y métodos}

\section{Localización del estudio}

El estudio se realizó en unidades de producción caprina de la comunidad de Xaltepec del municipio de Perote, ubicadas en la zona centro del estado de Veracruz, México. La comunidad se encuentra ubicada a $97^{\circ} 21^{\prime} 22.21^{\prime \prime}$ O y $19^{\circ} 22^{\prime} 50.06^{\prime \prime} \mathrm{N}$, a una altitud 2,358 msnm y colinda con el estado de Puebla; el clima es frío - seco, con temperatura media anual de $12{ }^{\circ} \mathrm{C}$ y precipitación media anual de $493.6 \mathrm{~mm}^{(23)}$.

La principal actividad pecuaria es la producción de caprinos y ovinos, bajo un sistema semiestabulado donde los propietarios y los familiares atienden a los animales. Los rebaños están integrados en promedio por 64 caprinos; los cuales en su mayoría pastorean terrenos comunales. En la temporada de mayor producción de forraje, algunos productores los confinan para utilizar los esquilmos agrícolas que se producen en la zona. Su principal producción es la leche para la elaboración de queso, y la carne a través de la venta de cabritos al destete y hembras de desecho ${ }^{(24)}$.

\section{Tipo de estudio y tamaño de muestra}

El estudio fue un ensayo clínico de fase III, realizado de septiembre de 2016 a marzo de 2018 para evaluar las tasas de parición, abortos y crías débiles en rebaños caprinos que resultaron positivos a brucelosis y que fueron vacunados con las cepas RB51-SOD, RB51 de Brucella abortus y Rev-1 de Brucella melitensis. Se estimó el tamaño de muestra a través del programa Win Episcope Ver. 2.0, con una prevalencia de $0.52 \%$ en cabras encontrada en un estudio previo en esa zona de Veracruz ${ }^{(24)}$, un intervalo de confianza de $95 \%$ y un error del 5\%. El tamaño mínimo de muestra fue de 72 cabras para cada grupo por tratamiento (cepa); cada bloque estuvo integrado por un subgrupo vacunado (36) y un subgrupo testigo (36). Cada grupo estudiado se integró con cabras mayores de tres meses, seronegativas a brucelosis y nunca vacunadas. Los animales seropositivos a brucelosis e identificados durante un muestreo inicial realizado previo a la vacunación para determinar los animales seropositivos, se mantuvieron en los rebaños para someterse a una exposición permanente en conjunto con los animales susceptibles. Los animales de cada grupo se identificaron con arete metálico en la oreja izquierda.

Para la realización de este trabajo se contó con la revisión del protocolo de investigación y la aprobación del mismo por la Comisión de Bioética de la Facultad de Medicina Veterinaria y Zootecnia de la Universidad Veracruzana. 


\section{Vacunación}

A los animales de los subgrupos vacunados de cada grupo, se les aplicaron $2 \mathrm{ml}$ de la vacuna por vía subcutánea en el lado izquierdo del tercio medio del cuello. Al primer grupo se le aplicó la cepa Rev-1 de Brucella melitensis a dosis de $1-2 \times 10^{9}$ UFC; el segundo con cepa RB51 de Brucella abortus, recibió $3 \times 10^{8}$ a $3 \times 10^{9}$ UFC y el tercero con RB51-SOD de Brucella abortus a dosis de $3 \times 10^{8}$ a $3 \times 10^{9}$ UFC; esta última es una vacuna importada por el Centro Nacional de Investigación Disciplinaria en Salud Animal e Inocuidad (CENID-SAI) del Instituto Nacional de Investigaciones Forestales, Agrícolas y Pecuarias (INIFAP) para investigación y proporcionada por el Dr. Gerhardt Shuring del Instituto Tecnológico de Virginia. Cada subgrupo vacunado tuvo su subgrupo testigo, animales que recibieron $2 \mathrm{ml}$ de solución salina fisiológica por la vía subcutánea en el lado izquierdo del tercio medio del cuello como placebo.

\section{Seguimiento de registros individuales}

Se establecieron registros individuales para cada uno de los animales integrantes de la muestra, de acuerdo con lo recomendado por el INIFAP para el manejo reproductivo de los caprinos en agostadero $^{(25)}$, con la finalidad de tener las fechas de los nacimientos, abortos y problemas presentados durante la gestación. Se realizó el cálculo de las tasas de gestación, pariciones y abortos para conocer la situación inicial de los rebaños, considerada como información de línea base para el estudio; asimismo, se dio seguimiento diario a los animales de la muestra por dos periodos de pariciones, para conocer el comportamiento posvacunación, así como indicadores como partos, abortos y nacimiento de crías débiles; las fechas correspondieron a la estacionalidad de pariciones que se tienen definidas en los rebaños (octubre - febrero).

\section{Cálculo de tasas de partos, abortos y pariciones de crías débiles}

Las tasas de partos, abortos y nacimiento de crías débiles en los tres grupos se integraron al utilizar la información incorporada en los registros individuales. Las diferencias entre grupos y la significancia de asociación, se realizó por análisis de datos categóricos $\left(\mathrm{Ji}^{2}\right)$ y el grado de asociación por Riesgo Relativo (RR) ${ }^{(26)}$.

\section{Resultados}

El Cuadro 1 muestra los indicadores productivos identificados en los rebaños caprinos en la comunidad de Xaltepec, del municipio de Perote, previo a la vacunación de los animales con las cepas del experimento, integrados por un total de 529 cabezas de caprinos. Las tasas 
promedio de gestación, pariciones y abortos fueron de $69.2,95.2$ y $2.7 \%$, respectivamente; esta información se consideró como línea base para los rebaños en estudio. Los rebaños utilizados presentaron una prevalencia general de brucelosis confirmada con la prueba de inmunodifusión radial (SRD) de $1.2 \%$ (IC95\%: 0.5-2.7) ${ }^{(27)}$.

Cuadro 1: Indicadores de inventario y reproductivos en rebaños de la comunidad de Xaltepec, Perote, Veracruz, México, previos a la vacunación

\begin{tabular}{lllll}
\hline \multirow{2}{*}{ Cepa } & $\begin{array}{l}\text { Inventario } \\
\text { (animales) }\end{array}$ & Tasa & & \\
\cline { 3 - 5 } & 134 & 67.5 & Pariciones & Abortos \\
\hline Rev -1 & 192 & 69.0 & 96.5 & 2.0 \\
RB51 & 203 & 71.3 & 95.6 & 3.0 \\
RB51-SOD & 529 & & & 3.3 \\
Total & & 69.2 & 95.2 & \\
Promedio & & & & 2.7 \\
\hline
\end{tabular}

Posterior a la vacunación, se evaluaron los indicadores de producción en los rebaños durante dos épocas de parición. En el Cuadro 2 se muestran las tasas de pariciones, abortos y nacimiento de crías débiles en el primer período de pariciones y se puede observar que los subgrupos vacunados tuvieron un comportamiento similar en relación a las tasas de partos y abortos; sin embargo, en los animales vacunados con la cepa Rev-1 se presentó una tasa de $5.5 \%$ (IC95\%: 0.9 - 20.0) de crías débiles, pero en los subgrupos vacunados con las cepas RB51 y con la RB51-SOD no se presentó esta condición. Los subgrupos controles mostraron un comportamiento similar a los subgrupos vacunados.

En el Cuadro 3 se muestra el Riesgo Relativo (RR) y la $\mathrm{Ji}^{2}$ de las pariciones, abortos y crías nacidas débiles del primer periodo de pariciones posvacunación y se aprecia que no hubo diferencia significativa $(P>0.01)$ entre los subgrupos vacunados y los subgrupos controles.

Cuadro 2: Indicadores de producción durante primer período de partos en rebaños caprinos vacunados con diferentes cepas en la comunidad de Xaltepec, Perote, Veracruz, México

\begin{tabular}{|c|c|c|c|c|c|c|c|c|c|c|c|}
\hline \multirow{2}{*}{ Cepa } & \multirow{2}{*}{ Grupo } & \multirow{2}{*}{$\mathbf{N}$} & \multicolumn{3}{|c|}{ Pariciones } & \multicolumn{3}{|c|}{ Abortos } & \multicolumn{3}{|c|}{ Crías débiles } \\
\hline & & & No & $\%$ & $\left(\mathrm{IC}_{95 \%}\right)$ & $\mathbf{N}$ & $\%$ & $\left(\mathrm{IC}_{95 \%}\right)$ & No & $\%$ & $\left(\mathrm{IC}_{95 \%}\right)$ \\
\hline \multirow{2}{*}{ Rev 1} & Vacunado & 36 & 24 & 66.6 & $48.9-80.9$ & 2 & 5.5 & $0.9-20.0$ & 2 & 5.5 & $0.9-20.0$ \\
\hline & Control & 36 & 22 & 61.1 & $43.5-76.3$ & 1 & 2.7 & $0.1-16.2$ & 0 & 0.0 & 0.0 \\
\hline \multirow{2}{*}{ RB51 } & Vacunado & 36 & 18 & 50.0 & $33.2-66.7$ & 1 & 2.7 & $0.1-16.2$ & 0 & 0.0 & 0.0 \\
\hline & Control & 36 & 21 & 58.3 & $40.8-74.0$ & 1 & 2.7 & $0.1-16.2$ & 0 & 0.0 & 0.0 \\
\hline RB51 & Vacunado & 36 & 25 & 69.4 & $51.7-83.0$ & 2 & 5.5 & $0.9-20.0$ & 0 & 0.0 & 0.0 \\
\hline$-\mathrm{SOD}$ & Control & 36 & 24 & 66.6 & $48.9-80.9$ & 1 & 2.7 & $0.1-16.2$ & 0 & 0.0 & 0.0 \\
\hline
\end{tabular}


Cuadro 3: Valores de Riesgo Relativo y $\mathrm{Ji}^{2}$ de pariciones, abortos y crías nacidas débiles del primer período de pariciones posvacunación

\begin{tabular}{llllllllll}
\hline \multirow{2}{*}{ Cepa } & \multicolumn{2}{l}{ Pariciones } & \multicolumn{3}{c}{ Abortos } & \multicolumn{3}{c}{ Crías débiles } \\
\cline { 2 - 10 } & $\mathbf{R R}$ & $(\mathbf{I C 9 5 \% )}$ & $\mathbf{J i}^{2}$ & $\mathbf{R R}$ & $\mathbf{( I C 9 5 \% )}$ & $\mathbf{J i}^{\mathbf{2}}$ & $\mathbf{R R}$ & $(\mathbf{I C 9 5 \%})$ & $\mathbf{J i}^{\mathbf{2}}$ \\
\hline Rev 1 & 0.4 & $0.1-1.3$ & 2.68 & 2.0 & $0.2-21.1$ & 0.4 & 0.9 & $0.1-2.9$ & 0.4 \\
RB51 & 0.4 & $0.1-2.0$ & 1.42 & 1.0 & $0.1-15.4$ & 0.0 & 0.0 & 0.0 & 0.0 \\
RB51- & 0.5 & $0.1-2.6$ & 0.73 & 2.0 & $0.2-21.1$ & 0.4 & 0.0 & 0.0 & 0.0 \\
SOD & & \multicolumn{4}{c}{$(P<0.01)}$. & & & &
\end{tabular}

En el segundo período de pariciones, se evaluó el comportamiento de los indicadores productivos de las hembras vacunadas y de los subgrupos control, los que se muestran en el Cuadro 4, donde se observa que los subgrupos vacunales en relación a la tasa de pariciones mejoraron el indicador en comparación con el primer periodo de pariciones. Los abortos sólo se presentaron en los animales vacunados con la cepa Rev-1 y en su grupo control, $2.7 \%$ (IC95\%: 0.1-16.2) y $5.5 \%$ (IC95\%: 0.9-20.0), respectivamente. Los animales vacunados con las cepas RB51 y RB51-SOD, no presentaron abortos; sin embargo, el grupo control de la cepa RB51 tuvo una tasa de $2.7 \%$ (IC95\%: 0.1-16.2). En los tres subgrupos vacunados, así como en los controles, no se presentaron casos de nacimiento de crías débiles. En el Cuadro 5, el análisis estadístico de estos resultados revela que no existe diferencia significativa entre los subgrupos vacunados y los controles $(P>0.01)$.

Cuadro 4: Indicadores de producción durante segundo período de partos en rebaños caprinos vacunados con diferentes cepas

\begin{tabular}{|c|c|c|c|c|c|c|c|c|c|c|c|}
\hline \multirow{2}{*}{ Cepa } & \multirow{2}{*}{ Grupo } & \multirow{2}{*}{$\mathbf{N}$} & \multicolumn{3}{|c|}{ Pariciones } & \multicolumn{3}{|c|}{ Abortos } & \multicolumn{3}{|c|}{ Crías débiles } \\
\hline & & & $\mathbf{N}$ & $\%$ & $\left(\mathrm{IC}_{95 \%}\right)$ & $\mathbf{N}$ & $\%$ & $\left(\mathrm{IC}_{95 \%}\right)$ & $\mathbf{N}$ & $\%$ & $\left(\mathrm{IC}_{95 \%}\right)$ \\
\hline \multirow{2}{*}{$\operatorname{Rev} 1$} & Vacunado & 36 & 33 & 91.6 & $76.4-97.8$ & 1 & 2.7 & $0.1-16.2$ & 0 & 0.0 & 0.0 \\
\hline & Control & 36 & 28 & 77.7 & $60.4-89.2$ & 2 & 5.5 & $0.9-20.0$ & 0 & 0.0 & 0.0 \\
\hline \multirow{2}{*}{ RB51 } & Vacunado & 36 & 34 & 94.4 & $79.9-99.0$ & 0 & 0.0 & 0.0 & 0 & 0.0 & 0.0 \\
\hline & Control & 36 & 31 & 86.1 & $69.7-94.7$ & 1 & 2.7 & $0.1-16.2$ & 0 & 0.0 & 0.0 \\
\hline RB51 & Vacunado & 36 & 34 & 94.4 & $79.9-99.0$ & 0 & 0.0 & 0.0 & 0 & 0.0 & 0.0 \\
\hline$-\mathrm{SOD}$ & Control & 36 & 32 & 88.8 & $73.0-96.3$ & 0 & 0.0 & 0.0 & 0 & 0.0 & 0.0 \\
\hline
\end{tabular}


Cuadro 5: Valores de Riesgo Relativo (RR) y $\mathrm{Ji}^{2}$ de pariciones, abortos y crías nacidas débiles del segundo período de pariciones posvacunación

\begin{tabular}{|c|c|c|c|c|c|c|c|c|c|}
\hline \multirow{2}{*}{ Cepa } & \multicolumn{3}{|c|}{ Pariciones } & \multicolumn{3}{|c|}{ Abortos } & \multicolumn{3}{|c|}{ Crías débiles } \\
\hline & $\mathbf{R R}$ & $(\mathrm{IC} 95 \%)$ & $\mathbf{J i}^{2}$ & $\mathbf{R R}$ & $\left(\mathrm{IC}_{95} \%\right)$ & $\mathbf{J i}^{2}$ & $\mathbf{R R}$ & $\left(\mathrm{IC}_{95} \%\right)$ & $\mathbf{J i}^{2}$ \\
\hline $\operatorname{Rev} 1$ & 0.3 & $0.1-1.3$ & 2.7 & 0.5 & $0.1-5.3$ & 0.4 & 0.0 & 0.0 & 0.0 \\
\hline RB51 & 0.4 & $0.1-1.9$ & 1.4 & 0.0 & 0.0 & 0.0 & 0.0 & 0.0 & 0.0 \\
\hline $\begin{array}{l}\text { RB51- } \\
\text { SOD }\end{array}$ & 0.5 & $0.1-2.6$ & 0.7 & 0.0 & 0.0 & 0.4 & 0.0 & 0.0 & 0.0 \\
\hline
\end{tabular}

$(P>0.01)$.

\section{Discusión}

En los rebaños del experimento, los animales seropositivos a la prueba serológica confirmatoria de inmunodifusión radial (SDR) para diagnóstico de brucelosis, permanecieron durante todo el período del estudio en sus rebaños de origen, para permitir el desafío directo natural de los animales vacunados y controles en los rebaños que contaron con una seroprevalencia general de $1.2 \%$ (IC $95 \%: 0.5-2.7)^{(27)}$, valor mayor al promedio general encontrado de $0.52 \%$ (IC95\%: 0.1 - 1.6) en 14 municipios de la zona centro del estado de Veracruz y al $0.05 \%$ que reporta el SENASICA a nivel nacional en rebaños caprinos ${ }^{(24)}$. La exposición de las hembras vacunadas dentro de los rebaños infectados permite desafiar la protección conferida en condiciones naturales; en este estudio, el desafío de los animales experimentales a la cepa de campo se evaluó a través de la seroprevalencia confirmada con la prueba $\mathrm{SRD}^{(27)}$. Para el desafío de rebaños experimentales en condiciones de campo es necesario considerar la seroprevalencia de la enfermedad detectada mediante pruebas más específicas (confirmatorias), porque de lo contrario no se garantiza el desafío de los rebaños vacunados con los animales seropositivos, ya que al usar solo una prueba tamiz, existe la posibilidad de tener animales falsos positivos considerados como infectados, y entonces la seropositividad puede deberse a la ventana que implica la seroconversión por el hecho mismo de la vacunación con cepas que tienen esta característica en esos animales o incluso por reacciones cruzadas con otros microorganismos y no permitir una correcta discriminación entre animales infectados y sólo reactores ${ }^{(16,17)}$.

En el Cuadro 1 se muestran las tasas de pariciones y abortos que en promedio son de $96.6 \%$ y $1.8 \%$, respectivamente; un comportamiento similar a lo señalado por otros investigadores en la caprinocultura de Oaxaca y Nuevo León, estados ubicados en las dos regiones que concentran $70.2 \%$ del inventario caprino nacional ${ }^{(4,28)}$; sin embargo, los rebaños caracterizados bajo condiciones de agostadero en el país registran una tasa de gestación menor a $65 \%$, lo que ocurre como producto de abortos, pobres condiciones sanitarias y severa restricción nutricional durante la gestación ${ }^{(11)}$. Al comparar los resultados de los 
subgrupos vacunados y controles con los indicadores observados en el presente estudio en la caprinocultura de Xaltepec, muestran que no es suficiente establecer programas de vacunación para prevenir o controlar enfermedades como la brucelosis, si no se incluye una alimentación adecuada de acuerdo con las características raciales de los animales que integran el rebaño y al sistema de producción, con la finalidad de mejorar la productividad de los mismos, además de que la mejora en los indicadores de pariciones, abortos o nacimiento de crías sanas en el primer periodo de pariciones posvacunación no es atribuible solo a la vacunación ${ }^{(11,13)}$.

Durante el primer período de partos posvacunación, la tasa de pariciones en todos los grupos que formaron parte del ensayo clínico, tanto vacunados como controles fue menor en comparación con los indicadores iniciales, debido a que no todas las hembras del experimento ingresaron al empadre; lo anterior pudo deberse a la edad, pobre condición corporal y a su estado nutricional; situación que coincide con los efectos que tiene la desnutrición durante el desarrollo y la vida postnatal temprana, ya que provocan efectos permanentes e irreversibles durante la pubertad, así como en la vida adulta de los pequeños rumiantes ${ }^{(13)}$. Los abortos se presentaron tanto en los subgrupos de animales vacunados con las cepas Rev-1 (B. melitensis), como con la RB51-SOD (B. abortus); en los subgrupos controles también se presentaron abortos con una tasa del $2.7 \%$ (IC95\%: 0.1 - 16.2); al compararse los grupos vacunados y controles, no se encontró diferencia significativa $(P<0.01)$.

Las tasas de nacimiento de crías débiles solo se presentaron en dos hembras que fueron vacunadas con la cepa Rev-1; es una condición que se puede presentar en las crías de animales infectados por brucelosis, situación que coincide con los resultados de serología realizada mediante la prueba de SDR donde dos hembras tuvieron serología positiva ${ }^{(6,27)}$. Las pérdidas fetales y abortos en los rebaños caprinos constituyen el principal problema reproductivo, que no solo se presenta por agentes infecciosos, sino también por el estrés nutricional de las cabras ${ }^{(11)}$. Aunado a ello, la desnutrición afecta a los animales expuestos a las cepas vacunales o de campo, al provocar que el animal no produzca anticuerpos mesurables por las pruebas serológicas convencionales o no establezca una protección contra el agente causal ${ }^{(29-33)}$.

Durante el segundo período de partos después de la vacunación, las tasas de pariciones observadas en los tres grupos mostraron un comportamiento similar; sin embargo los abortos se presentaron en los subgrupos vacunal y control de la capa Rev-1, $5.5 \%$ (IC $95 \%$ : 0.9 - 20.0) y en el subgrupo control de la RB51, $2.7 \%$ (IC95\%: 0.1 - 16.2); ningún subgrupo vacunado tuvo nacimiento de crías débiles; al realizar el análisis estadístico se observó que no hubo diferencia significativa $(P<0.01)$; cabe señalar que la condición de vacunación no está asociada con la presencia del aborto. Villa et $l^{(17)}$ realizaron la evaluación de vacunas para el control de la brucelosis y encontraron que la cepa RB51 (B. abortus) tuvo una tasa de 
abortos de $74.2 \%$, el cual se considera alto, además de presentar el mayor riesgo relativo de presentar abortos en comparación con resultados de otros estudios en los que se vacunaron con esa cepa cabras y borregas gestantes y se encontraron tasas de abortos menores a $10 \%(29,31,33)$; por ello no se recomienda utilizarla en cabras. Sin embargo, esta información difiere de lo encontrado en este estudio en la comunidad de Xaltepec ya que, de acuerdo con otros estudios, se encontró que su aplicación en pequeños rumiantes puede ocasionar hasta el $1 \%$ de abortos en las hembras susceptibles por efecto vacunal ${ }^{(31)}$. De este modo, es recomendable vacunar hembras mayores de tres meses y no gestantes, condición que es necesario cumplir para evitar el riesgo de abortos por efecto vacunal, situación que algunos países han establecido como norma para evitar pérdidas económicas ${ }^{(18)}$. La cepa vacunal RB51 ( $B$. abortus), está autorizada para su uso en México solo para la especie bovina; sin embargo, la autoridad sanitaria en el año 2005 registró una vacuna con estas características para uso en cabras ${ }^{(34)}$ y que coincide con este estudio, en el sentido de que la cepa es segura porque no produjo abortos en las hembras del subgrupo correspondiente en la comunidad de Xaltepec.

Existe información que señala que se puede presentar hasta un $2 \%$ de abortos en las hembras susceptibles por efecto vacunal ${ }^{(14,31)}$; sin embargo, es importante considerar que existen factores propios de cada individuo susceptible como son edad, sexo, estado reproductivo, condición inmunitaria y nutricional, así como del agente, que alteran el desarrollo de la protección que genera la vacuna y en general, la protección que confieren las vacunas contra brucelosis la cual oscila entre 85 y $90 \%{ }^{(30,32,35)}$. El nacimiento de crías débiles que mueren en el peri-parto es una condición que se puede presentar en las crías de animales infectados por brucelosis, situación que coincide con los resultados de serología realizada mediante la prueba de SDR donde dos hembras tuvieron serología positiva ${ }^{(6,27)}$.

Las cepas Rev-1 (B. melitensis) y RB51 (B. abortus) son cepas que han sido evaluadas en su protección, así como en sus efectos colaterales en los animales vacunados. En la actualidad se han desarrollado vacunas de plásmidos de ADN las que representan una alternativa de vacuna de sobreexpresión homóloga como la cepa RB51-SOD (CU/Zn) utilizada en este estudio, la que en modelos murinos presenta una protección mejor contra $B$. abortus que la establecida por la RB51 ${ }^{(19)}$ y se considera dentro de las vacunas de ADN que demuestran capacidad de producir inmunidad celular, humoral y cierto grado de inmunidad protectora $^{(16,31,33)}$. En bovinos se sugiere la producción de anticuerpos y CMI de tipo Th-1, y genera protección en los animales vacunados al desafío con $B$. abortus ${ }^{(19)}$. Al evaluar el comportamiento de la cepa RB51 - SOD en campo en rebaños caprinos con seroprevalencias del $1.2 \%$ (IC $95 \%$ : 0.5 - 2.7), los animales vacunados con esta cepa no presentaron aborto, ni nacimientos de crías débiles. Es importante señalar que de acuerdo con resultados publicados, los animales vacunados con esta cepa no seroconvierten a las pruebas convencionales a los 90 días posvacunación, por lo cual tampoco genera confusión diagnóstica ${ }^{(27)}$. 
Respecto al efecto que tiene sobre el incremento del nacimiento de crías, no hubo diferencia significativa $(\mathrm{P}<0.01)$ entre las cabras vacunadas con las cepas Rev-1 (B. melitensis) y RB51 (B. abortus). Olsen et $a l^{(24)} l$, al evaluar la cepa RB51-SOD en bisontes y compararla con la cepa RB51, no encontraron diferencias en el comportamiento de ambas cepas, y sugieren que los datos obtenidos para la cepa RB51 - SOD es segura para esa especie, ya que no se observa la presencia del agente vacunal en tejidos; sin embargo, los resultados de la eficacia vacunal recomiendan que la cepa RB51 sería preferible a la RB51-SOD para la vacunación de crías de bisonte ${ }^{(24)}$; no obstante, es necesario evaluar su condición del desarrollo de la inmunidad de tipo celular y humoral en las cabras; así como su eficacia y seguridad en animales jóvenes y adultos.

\section{Conclusiones e implicaciones}

Al evaluar las tasas de pariciones, abortos y nacimiento de crías débiles en rebaños vacunados con la cepa RB51 - SOD (B. abortus) no se tuvo diferencia significativa entre los animales inoculados con la Rev-1 (B. melitensis) y la RB51 (B. abortus), cepas disponibles para uso en las Campañas Zoosanitarias oficiales, así como con los subgrupos control; lo que sugiere que la cepa RB51 - SOD puede generar beneficios similares en la protección contra la enfermedad en el rebaño para mantener el inventario sano y evitar los efectos negativos sobre la vida productiva, la salud de las hembras, así como garantizar la sanidad del rebaño; sin embargo, es necesario considerar actividades complementarias de manejo y alimentación del rebaño para mejorar condiciones productivas de los animales susceptibles.

\section{Agradecimientos y conflictos de intereses:}

Al Programa de Doctorado en Ciencias Agropecuarias de la Universidad Veracruzana y al CONACYT por la oportunidad recibida para desarrollar los estudios de Doctorado en Ciencias del primer autor; así también al CENID-SAI del Instituto Nacional de Investigaciones Forestales, Agrícolas y Pecuarias por la importación de la vacuna RB51SOD y al Dr. Gerhardt Shuring del Instituto Tecnológico de Virginia por donarla para el estudio. A los productores de la comunidad de Xaltepec, municipio de Perote, Veracruz México, por las facilidades otorgadas para realizar el presente experimento en sus unidades de producción caprina.

\section{Literatura citada:}

1. Dean AS, Crump L, Greter H, Schelling E, Zinsstag J. Global burden of human brucellosis: a systematic review of disease frequency. PLoS Negl Trop Dis 2012;6(10):1865. http://dx.doi.org/10.1371/journal.pntd.0001865. 
2. Moreno E. Retrospective and prospective perspectives on zoonotic brucellosis. Front Microbiol 2014;(5):213.

3. Godfroid J, Scholz HC, Barbier T, Nicolas C, Wattiau P, Fretin D, Saegerman C. Brucellosis at the animal ecosystem human interface at the beginning of the 21 st century. Prev Vet Med 2011;102(2):118-131.

4. SAGARPA - Comité Nacional Sistema Producto Caprinos (CNSPC). Plan Anual de Fortalecimiento. México, D.F. htttp://www.cnsp.caprinos.org.mx. 2015.

5. Ducrotoy MJ, Conde-Álvarez R, Blasco JM, Moriyón I. A review of the basis of the immunological diagnosis of ruminant brucellosis. Vet Immunol Immunopathol 2016;171:81-102.

6. Blasco JM. Control and eradication strategies for Brucella melitensis infection in sheep and goats. Prilozi 2010;31(1):145-165.

7. Guzmán-Hernández RL, Contreras-Rodríguez A, Ávila-Calderón ED, Morales-García MR. Brucelosis: zoonosis de importancia en México. Rev Chilena Infect 2016;33(6):656-662.

8. Montiel DO, Bruce M, Frankena K, Udo H, Van DZA, Rushton J. Financial analysis of brucellosis control for small-scale goat farming in the Bajío region, Mexico. Prev Vet Med 2015;118(4):247-259.

9. Robles C. Sanitary aspects in small ruminants in extensive systems in South America. Rev Arg Prod Anim 2017;37(1):5-8.

10. Cantú CA, Álvarez OG, Zapata CCC. Situación epidemiológica de las principales enfermedades en cabras. Avances en la producción de pequeños rumiantes en el noreste de México. Ediciones UAT. 2015:55-56.

11. Mellado M, Olivares L, López R, Mellado J. Influence of lactation, liveweight and lipid reserves at mating on reproductive performance of grazing goats. J Anim Vet Ad 2005;4(4):420-423.

12. SAGARPA. Norma Oficial Mexicana NOM-041-ZOO-1995, Campaña Nacional contra la Brucelosis en los Animales. SAGARPA: Secretaría de Agricultura Ganadería y Desarrollo Rural, México. 1996.

13. Mellado M. Técnicas para el manejo reproductivo de las cabras en agostadero. Trop Subtrop Agroecosys 2008;9(1):47-63.

14. Blasco JM, Molina FB. Control and eradication of Brucella melitensis infection in sheep and goats. Vet Clin Food Anim 2011;(27):95-104. 
15. Menzies PI. Vaccination programs for reproductive disorders of small ruminants. Anim Reprod Sci 2012;130(3):162-172.

16. Dorneles EM, Sriranganathan N, Lage AP. Recent advances in Brucella abortus vaccines. Vet Res 2015;46(1):76.

17. Villa R, Perea M, Díaz AE, Soberón MA, Hernández AL, Suárez GF. Abortions and stillbirths in goats immunized against brucelosis using RB51, rfbK and Rev 1 vaccines. Téc Pecu Méx 2008;46(3):249-258

18. Martínez HDI, Morales MJA, Peniche CAE, Molina SB, Rodríguez CMA, Loeza LR, Flores-Castro R. Use of RB51 Vaccine for small ruminants Brucellosis prevention, in Veracruz, México. Int J Dairy Sci 2010;(5):10-17.

19. Oñate AA, Céspedes S, Cabrera A, Rivers R, González A, Muñoz C, Folch H. DNA vaccine encoding $\mathrm{Cu}, \mathrm{Zn}$ superoxide dismutase of Brucella abortus induces protective immunity in BALB/c mice. Infect Immun 2003;71(9):4857-4861.

20. Solorio-Rivera JL, Segura-Correa JC, Sánchez-Gil LG. Seroprevalence of and risk factors for brucelosis of goats in herds of Michoacan, Mexico. Prev Vet Med 2007;(82):282-290.

21. Olsen SC. Recent developments in livestock and wildlife brucellosis vaccination. Rev Sci Tech 2013;32(1):207-17.

22. Olsen SC, Boyle SM, Schurig GG, Sriranganathan NN. Immune responses and protection against experimental challenge after vaccination of bison with Brucella abortus strain RB51 or RB51 overexpressing superoxide dismutase and glycosyltransferase genes. Clin Vaccine Immunol 2009;16(4):535-540.

23. INEGI. Anuario estadístico y geográfico de Veracruz de Ignacio de la Llave. 2016.

24. Román-Ramírez DL, Martínez-Herrera DI, Villagómez-Cortés JAJ, Peniche-Cardeña AE, Morales-Álvarez JF, Flores-Castro R. Epidemiología de la brucelosis caprina en la Zona Centro del Estado de Veracruz. Gac Med Mex 2017;153(1):26-30.

25. Raúl AC, Clemente LCJ, Ivone MPM, Denis OAJ, Melesio SH. Manejo reproductivo de los caprinos en agostaderos de B.C.S. INIFAP. 2009.

26. Thrusfield M. Veterinary epidemiology. 3ra ed. USA: Blackwell Science Ltd; 2005. 
27. Molina SB, Martínez HDI, Pardío SVT, Flores CR, Morales AJF, Murguía GJ, et al. Evaluación de la seroconversión en cabras vacunadas con diferentes cepas contra la brucelosis en Veracruz, México. Avances en Investigación Agrícola, Pecuaria, Forestal, Acuícola, Pesquería, Desarrollo Rural, Transferencia de tecnología, Biotecnología, Ambiente, Recursos naturales y Cambio climático 2017;1(1):810-819.

28. Banda CA. Seroprevalencia de brucelosis y su efecto sobre la productividad de hatos caprinos en Aramberri, Nuevo León [tesis doctorado]. México: Universidad Autónoma de Nuevo León. 2015.

29. Ochoa DV. Protección conferida por la vacunación con Rev 1 Brucella melitensis; RB51 y rfbK Brucella abortus, en borregas desafiadas experimentalmente con Brucella melitensis [tesis maestría]. México: Universidad Nacional Autónoma de México; 2002.

30. Mellado M, Olivares L, Díaz H, Villarreal JA. Placental traits in pen-fed goats and goats kept on rangeland. J App Anim Res 2006;29(2):133-136.

31. Moriyón I, Grilló MJ, Monreal D, González D, Marín C, López-Goñi I, Blasco JM. Rough vaccines in animal brucellosis: structural and genetic basis and present status. Vet Res 2004;35(1):1-38.

32. Estein SM. Brucelosis bovina (revisión bibliográfica) Revista Electrónica de Veterinaria $\begin{array}{llllll}\text { REDVET 2013. } & \text { ISSN } & 1695 & - & 7504, & 7(5) .\end{array}$ http://www.veterinaria.org/revistas/redvet/n050506.html

33. El Idrissi AH, Benkirane A, El Maadoudi M, Bouslikhane M, Berrada J and Zerouali A. Comparison of the efficacy of Brucella abortus strain RB51 and Brucella melitensis Rev 1 live vaccines against experimental infection with Brucella melitensis in pregnant ewes. Rev Sci Tech Off Int Epiz 2001;20(3):741-747.

34. SENASICA. Dirección General de Salud Animal. Regulación y Registro de Productos Veterinarios. Lista de Productos Biológicos 2019. https://www.gob.mx/cms/uploads/attachment/file/455887/LISTADO_PRODUCTOS_ BIOLOGICOS_2019.pdf

35. Schurig GG, Sriranganathan N, Corbel M J. Brucellosis vaccines: past, present and future. Vet Microbiol 2002;(90)479-496. 